\title{
STRUCTURAL AND MAGNETIC DYNAMICAL PROPERTIES OF SOLIDS. EXPERIMENTAL APPROACH WITH INELASTIC NEUTRON SCATTERING
}

\author{
B. HENNION \\ Laboratoire Léon Brillouin, CEA-CNRS \\ CE Saclay, 91191 Gif sur Yvette Cedex, France
}

Inelastic neutron scattering on single crystals is a privileged way to obtain valuable information on dynamical properties of solids. The basis of the technique is summarised. Master formulae and experimental examples for lattice dynamics and spin dynamics studies are presented.

PACS numbers: $63.20 .-\mathrm{e}, 63.20 . \mathrm{Kr}, 75.30 . \mathrm{Ds}, 75.40 . \mathrm{Gb}, 74.25 . \mathrm{Ha}$

\section{Introduction}

The understanding of many aspects of solids necessitates a good knowledge of their dynamical properties. Indeed, in the range of conditions usually implied in solid state physics, most phenomena are related to the way that a perturbed system uses to relax the perturbation. In the framework of the linear response theory, the relevant quantity is the dynamical susceptibility $\chi_{A B}(\omega)$, time Fourier transform of the thermal average of the response function $\Phi_{A B}(t)$, where $A$ is the variable associated to the operator $\widehat{B}$, which provides the perturbation at time 0 . When a system possesses translational invariance, the relevant quantities are described in terms of plane waves, the Bloch states.

The dynamical response of an ordered system to a perturbation involves collective excitations, which are phonons for the structural properties and spin waves for the magnetic properties. These excitations depend on the microscopic parameters which are responsible for the ordered state, and their measurements provide direct information on these parameters. Concerning structural properties, ultrasonic measurements and light scattering measurements give access to sound velocities and to optic modes at $q=0$. Concerning magnetic properties, light scattering, in some cases, makes it possible to determine the gap of the spin wave spectrum. X-ray scattering could be used, in principle, to measure phonons and eventually spin waves. But the energy range of $\mathrm{X}$ rays is of several $\mathrm{keV}$, which precludes accurate determination of phonon or magnon energies which are in the 
range of several meV. Neutron scattering is the only technique which allows a complete determination of the phonon- or spin-wave spectra.

The spatial correlations between atoms or magnetic moments give rise to a $\boldsymbol{q}$-dependence of the collective excitations. To get an accurate determination of phonons or spin waves, it is therefore necessary to work on single crystals. The triple-axis spectrometer (TAS) is the instrument which is devoted to the determination of inelastic neutron scattering by single crystals.

In Sec. 2 the general principle of the spectrometer will be presented. Section 3 will be devoted to phonon scattering and illustrated by a few examples to present as well the general features of lattice dynamics as the more specific example of a charge density wave system: the blue bronze. The case of spin dynamics will be introduced in Sec. 4 and exemplified with the measurement of the magnetic excitations of the spin-Peierls compound $\mathrm{CuGeO}_{3}$. The very singular magnetic dynamical response of high- $T_{\mathrm{c}}$ systems will be also presented.

\section{Inelastic neutron scattering on TAS spectrometer}

The principle of the triple-axis spectrometer, invented by B.N. Brockhouse, is very simple and is schematized in Fig. 1. Neutrons of selected wave vector $k_{1}$ are sent on a sample and the scattered beam is analysed in order to select neutrons of final wave vector $k_{\mathrm{f}}$. Any neutron which is then detected has exchanged momentum and energy with the sample. Momentum and energy transfers are given by the conservation laws

Neutron source
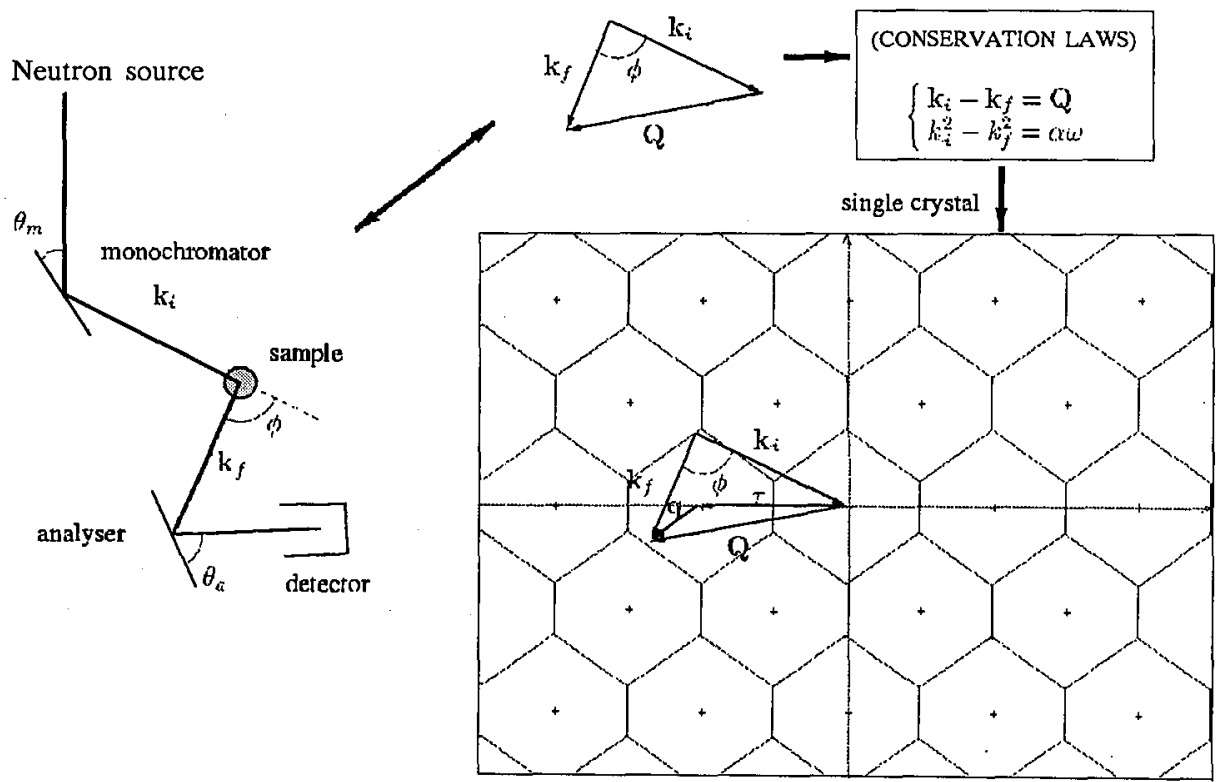

Fig. 1. Principle of a triple-axis spectrometer: to a selected configuration of the spectrometer corresponds a single $(\boldsymbol{Q}, \omega)$ point. For a periodic lattice, the projection in the reciprocal space of the sample yields a single $(q, \omega)$ point, with $Q=\tau+q$. 


$$
\left\{\begin{array}{r}
\hbar k_{\mathrm{i}}-\hbar k_{\mathrm{f}}=\hbar \boldsymbol{Q}, \\
\hbar^{2} k_{\mathrm{i}}^{2} / 2 M_{n}-\hbar^{2} k_{\mathrm{f}}^{2} / 2 M_{n}=\hbar \omega,
\end{array}\right.
$$

the scattering angle completing the vectorial definition of the momentum transfer.

Scattering of neutrons by a sample also means that the physics of the sample induces non-zero value for its dynamical susceptibility $\chi(Q, \omega)$. In the simplest case of a perfectly ordered system, these non-zero values correspond to collective excitations, which are quasi-particles such as phonons or spin waves. Because of translational invariance, their wave vectors are defined in the first Brillouin zone and noted $\boldsymbol{q}$ and the momentum transfer $\boldsymbol{Q}$ has to be decomposed in $\boldsymbol{Q}=\boldsymbol{\tau}+\boldsymbol{q}$, where $\tau$ is a reciprocal lattice vector.

The realisation of the instrument implies a neutron source with a constant flux. The selection of $k_{\mathrm{i}}$ and $k_{\mathrm{f}}$ is done by using the Bragg reflections on single crystals, respectively monochromator and analyser. A position of the spectrometer is fixed by the choice of $k_{\mathrm{i}}, k_{\mathrm{f}}$ and the scattering angle. To this position corresponds a single point $(\boldsymbol{Q}, \omega)$ in the relevant space of the sample dynamics. But it is easy to check that a selected $(Q, \omega)$ point may be obtained by a whole set of $k_{\mathrm{i}}$ and $k_{\mathrm{f}}$ values. Scanning the $Q, \omega$ space step by step to collect scattered intensity makes it possible to determine the spectrum of phonons or spin waves, in an energy range typically from 0.1 to $100 \mathrm{meV}$.

The scattered intensity is proportional to the inelastic neutron scattering cross-section, which is specific of the dynamics of the studied system. We will see later on what are the formulae for phonon and spin wave processes. The common feature is that measurements on triple-axis spectrometer imply partial differential scattering cross-sections and the compensation of getting minute details of the dynamical response is a low counting rate. To overcome this difficulty the possibilities are somewhat limited.

- The available neutron flux in the energy range under consideration depends on neutron sources. Steady reactors have nearly reached their optimum in the 1970's with the high flux reactor of the Institute Laue-Langevin (ILL, Grenoble, France) with a constant thermal flux of $1.5 \times 10^{15}$ neutrons/( $\left.\mathrm{cm}^{2} \mathrm{~s}\right)$ for $57 \mathrm{MW}$ of thermal power. Nevertheless, the optimisation of the use of the available neutron beams made it possible to gain nearly a factor of ten on the scattered intensity, thanks to the development of focusing devices.

Spallation sources are still developing and the European Spallation Source project could provide one order of magnitude more neutrons than current sources as e.g. ISIS (Rutherford Laboratory, Great Britain). But this gain is conditioned by the use of a pulsed source, the benefit of which is essentially restricted to time-of-flight measurements, which in most cases are inadequate to work on single crystals.

- When possible, the easiest way to enhance the scattered intensity is to use a single crystal as large as possible. The neutron absorption is usually low or may be decreased by the use of specific isotopes, so that centimetre size samples can be used very efficiently. The numbers to keep in mind are: (1) most measurements may be carried out on samples of a few hundreds of $\mathrm{mm}^{3},(2)$ in some favorable cases a 
few $\mathrm{mm}^{3}$ are enough to obtain valuable information (e.g. single grain quasicrystals, $\mathrm{C}_{60}, \mathrm{MBE}$ grown samples).

- Finally, the experimentalist has to adjust the resolution of the triple-axis spectrometer, to find the best compromise between the accuracy of the measurement and a realistic counting rate. Both are related to the wave vectors of incident and scattered neutrons and to the angular variables of the spectrometers (angular divergences of the beam and mosaicities of monochromator, analyser, and sample). This is due to the use of the Bragg scattering to select neutron wave vectors, and it means that when the spectrometer defines a $\left(\boldsymbol{Q}_{0}, \omega_{0}\right)$ point, the measurement consists indeed in an integration in $Q, \omega$ space around this point with a weighting corresponding to the resolution function of the spectrometer, so that the measured intensity is $I\left(\boldsymbol{Q}_{0}, \omega_{0}\right)=\int R\left(\boldsymbol{Q}-\boldsymbol{Q}_{0}, \omega-\omega_{0}\right) S(\boldsymbol{Q}, \omega) \mathrm{d}^{3} \boldsymbol{Q} \mathrm{d} \omega$, and to get the physics out of the neutron data it is necessary to take this effect into proper account. With classical approximations by Gaussian laws for collimator transmission and mosaic distribution, the resolution function may be expressed as a 4-dimensional Gaussian distribution centered on $\left(\boldsymbol{Q}_{0}, \omega_{0}\right): R(\boldsymbol{X})=R_{0} \exp \left(-\boldsymbol{X}^{\mathrm{T}} A \boldsymbol{X}\right)$, where $\boldsymbol{X}=\left[\boldsymbol{Q}-\boldsymbol{Q}_{0}, \omega-\omega_{0}\right]$. The factor $R_{0}$, which weights the scattered intensity, and the matrix $A$, which yields the accuracy of the measurement, through the convolution with the scattering law, are both related to $k_{\mathrm{i}}, k_{\mathrm{f}}$ and to angular variables of the spectrometer [1]. The common sense is largely obeyed, which indicates that improving the accuracy leads to a decrease in the scattered intensity. Due to more subtle effects related to the convolution, focusing effects may arise and provide an optimal choice in some cases.

\section{Lattice dynamics}

The master formula of the 1-phonon coherent inelastic neutron scattering law for a harmonic phonon process may be found in Ref. [2] and may be written as

$$
\begin{aligned}
& S(\boldsymbol{Q}, \omega)=\frac{k_{\mathrm{f}}}{k_{\mathrm{i}}} \frac{(2 \pi)^{3}}{v_{0}} \sum_{\tau} \sum_{j, \boldsymbol{q}} \delta(\boldsymbol{Q}+\boldsymbol{q}-\boldsymbol{\tau})\left|F_{\boldsymbol{q}}^{j}(\boldsymbol{Q})\right|^{2} \\
& \times \frac{1}{2 \omega_{j}(\boldsymbol{q})}\left\{n_{j}(\boldsymbol{q}) \delta\left[\omega+\omega_{j}(\boldsymbol{q})\right]+\left[1+n_{j}(\boldsymbol{q})\right] \delta\left[\omega-\omega_{j}(\boldsymbol{q})\right]\right\},
\end{aligned}
$$

where $\left|F_{q}^{j}(Q)\right|^{2}$ is called the dynamical structure factor, with

$$
F_{\boldsymbol{q}}^{j}(\boldsymbol{Q})=\sum_{l} \bar{b}_{l} \exp \left[-W_{l}(\boldsymbol{Q})\right] \exp \left(-\mathrm{i} \boldsymbol{Q} \cdot \boldsymbol{r}_{l}\right)\left[\boldsymbol{Q} \cdot \boldsymbol{\xi}_{l}^{j}(\boldsymbol{q})\right] M_{l}^{-1 / 2}
$$

$l$ indices different atoms at positions $r_{l}$ in the primitive cell of volume $v_{0}$, $\bar{b}_{l}$ are the coherent scattering lengths, averaged on the isotopic composition, and $W_{l}(\boldsymbol{Q})$ are the partial Debye-Waller coefficients. $j$ indices different eigenstates of the dynamical matrix, $\xi^{j}(q)$ - the corresponding eigenvectors, and $n_{j}(q)$ are the Bose thermal factors, namely $1 /\left[\exp \left(\frac{\hbar\left|\omega_{j}(q)\right|}{k_{B} T}\right)-1\right]$. When phonons are damped, $\delta\left[\omega-\omega_{j}(q)\right]$ is replaced by the spectral weight function, which is often described as a damped harmonic oscillator.

Inelastic neutron scattering provides information on: (1) the dispersion of phonon branches (the eigenvalues of the dynamical matrix of the system), 
(2) the strength of the scattering, which involves the eigenvectors, (3) the eventual damping of the phonons, due for instance to anharmonicity or to electron-phonon interaction. It may be noticed that the scalar product between the momentum transfer and the polarisation vector of a phonon mode provides a good test for the identification of the mode, comparing the scattered intensities obtained in different Brillouin zones.

The information on the eigenvalues and eigenvectors yield a very valuable check of lattice dynamics models and this has been widely used. But another point of view is that in many cases, anomalies in the phonon spectrum are the signature of structural or electronic properties which can thus be revealed by inelastic neutron scattering. This is the case for structural phase transitions where precursor dynamical effects may be observed. On the other hand, neutron scattering does not give direct information on charges, but when an electron-phonon coupling induces anomalies in the phonon spectrum, the evolution of a particular part of the phonon spectrum may be a very good way to follow the evolution of electronic properties.

\subsection{Determination of a phonon spectrum}

In the early age of neutron scattering, triple-axis spectrometry has been largely used to determine phonon spectra in various compounds. The goal was on the one hand to understand structural properties and on the other hand to ascertain models able to describe lattice dynamics and to account for the experimental data. The basis of these models was a description in terms of short-range force constants and long-range Coulomb forces. The Born-von Karman model, the rigid ion model, the valence force field model, and various shell models have been developed and found relevant for specific families of compounds.

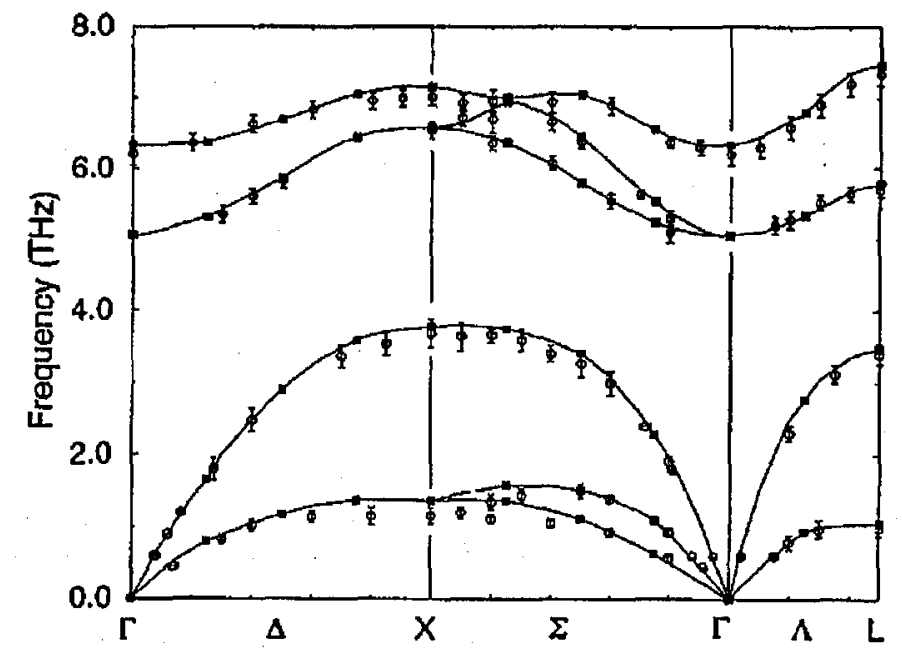

Fig. 2. Phonon spectrum of $\mathrm{CuCl}$. Filled squares and solid lines are from $a b$ initio calculations [3]. Open circles are measured data at $4.2 \mathrm{~K}$ [4]. 
Due to the development of computing facilities, the current tendency is to use $a b$ initio calculations to solve the structural properties of compounds, including their phonon spectrum. An example of this trend is illustrated in Fig. 2 for the case of $\mathrm{CuCl}$. For this ionic compound, with zinc-blende structure, there are 6 phonon branches. Measurements of phonon modes in the main symmetry directions, reported as open circles in Fig. 2, allowed the refinement of a shell model to account for the observed lattice dynamics and adjusted parameters may be found in [3] and [4]. But now the filled squares and solid lines of Fig. 2 are indeed deduced from $a b$ initio calculations [5]. The nice agreement emphasises the quality of current $a b$ initio calculations but also points out the need for clean experimental data which could be used to validate such calculations.

\subsection{Electron-phonon coupling and Peierls transition in a charge density wave system, the blue bronze}

In many cases the number of atoms per primitive unit cell precludes a complete determination of the phonon spectrum. Nevertheless, quite often only a restricted part of the phonon spectrum is needed to get access to the relevant physical parameters. This is generally the case of structural phase transitions. To illustrate what can then be done with neutron scattering, we will consider the case of the Peierls transition, which is associated with an electron-phonon coupling in a quasi-one-dimensional conductor, responsible for the instability of a ID metallic chain against charge density waves.

The electron-phonon coupling induces anomalies in the phonon spectrum, the so-called Kohn anomalies, at $\boldsymbol{q}$ positions related to the wave vector of the electrons at the Fermi energy and, as long as the system has not undergone a transition from its high-temperature metallic state to its low-temperature semiconductor state, these anomalies can be directly observed only by inelastic neutron scattering on single crystals. Figure 3 displays the evolution of the inelastic neutron scattering in the vicinity of the Kohn anomaly in blue bronze $\left(\mathrm{K}_{3} \mathrm{Mo}_{10} \mathrm{O}_{30}\right)$, where a Peierls transition occurs at $183 \mathrm{~K}$ [6]. Above the transition the Kohn anomaly is seen as a dip on a particular phonon branch (at $q=\left(1,2 k_{\mathrm{f}},-0.5\right)$ in that case). The softening and damping of this phonon increase when the temperature is lowered, leading to a structural transition towards an incommensurate phase, associated with the loss of the metallic character of the system. Below the transition, specific excitations (phason and amplitudon modes) related to the incommensurate phase are observed.

The evolution of the Kohn anomaly and the characteristics of the phason and amplitudon modes are directly related to electronic parameters of the system and could be deduced from the neutron scattering measurements. Figure 4a shows the overall evolution of the frequencies and damping of the Kohn anomaly above the transition and of the phason and amplitudon modes below it. The values have been deduced from a fit of neutron data, using a damped harmonic oscillator description for all excitations [6]. Below the transition the phase mode has a linear dispersion and the phason velocity evolves with temperature as a function of the Peierls gap. This could be checked down to $100 \mathrm{~K}$ [7] as illustrated in Fig. 4b. 


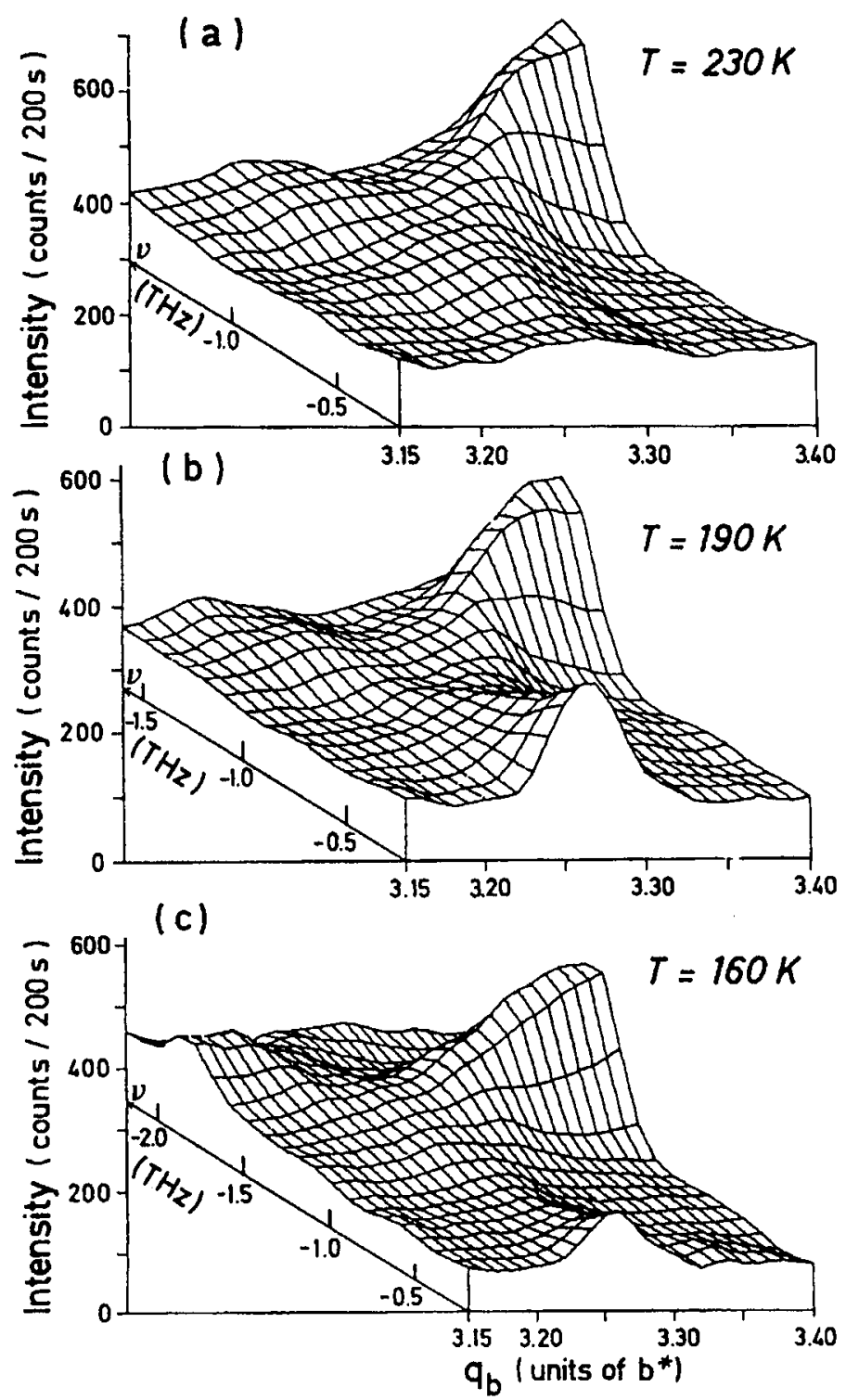

Fig. 3. Evolution with temperature of the scattered intensity on a satellite position $\left(q_{b} \approx 3.27\right)$ of the incommensurate low temperature phase of blue bronze [6].

But a further conclusion of this neutron scattering study is the inadequacy of a mean field theory to describe the observed behaviour of the dynamics near the Peierls transition. Indeed just below the transition, the amplitude mode and the phase mode should start from the same frequency, which should be equal to 

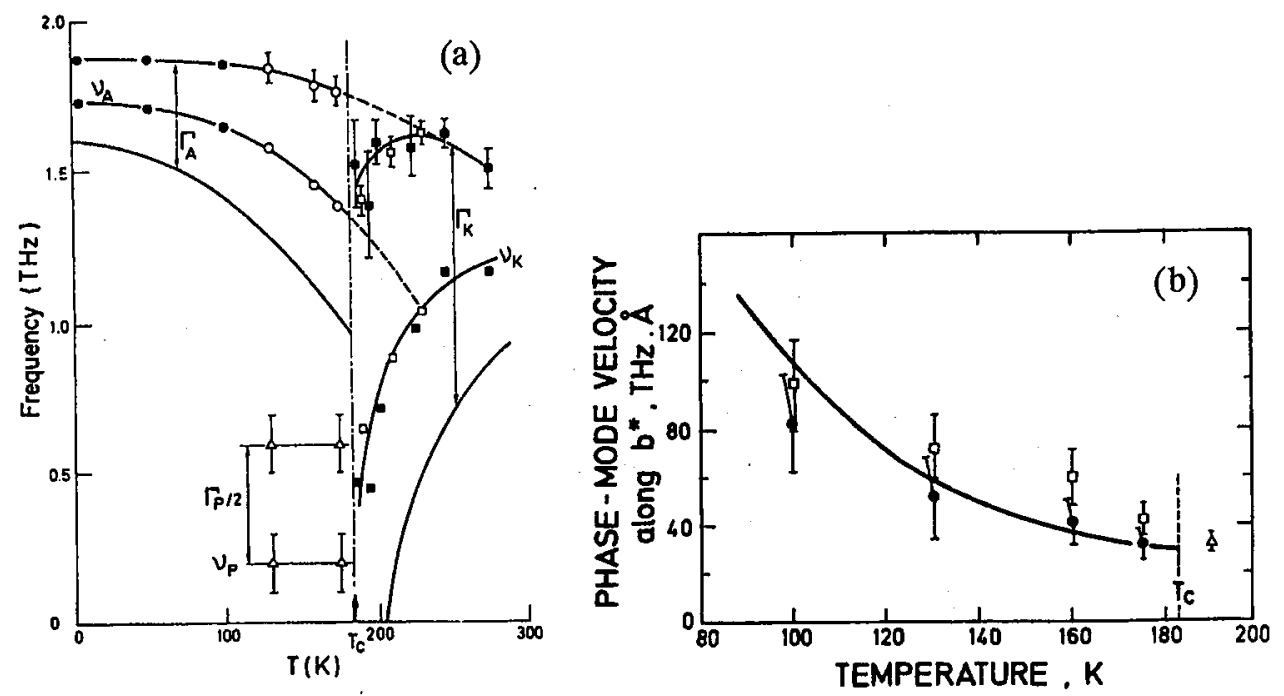

Fig. 4. (a) Temperature dependence of the quasiharmonic frequency $\nu_{i}$ and the frequencies $\nu_{i} \pm \Gamma_{i} / 2$ for the Kohn anomaly $(i=\mathrm{K}$, open and filled squares correspond to 2 different sets of measurements), amplitude mode ( $i=A$, open circles) and phase mode ( $i=\mathrm{P}$, open triangles) of blue bronze at $2 k_{\mathrm{f}}$, including Raman scattering data (filled circles) from Ref. [9] for $\nu_{\mathrm{A}}$ and $\Gamma_{\mathrm{A}}$ at and below $100 \mathrm{~K}$; (b) Thermal evolution of the phase mode velocity in the $b^{*}$ direction neglecting $(\square)$ or accounting for $(\bullet)$ the anisotropy of the surface dispersion. $\Delta$ is deduced from the curvature of the Kohn anomaly at $190 \mathrm{~K}[7]$.

that of the Kohn anomaly just above the transition. The data reported in Fig. $4 \mathrm{a}$ clearly show that this is not the case. An alternative approach using molecular dynamics simulation showed a much nicer agreement [8].

\section{Spin dynamics}

The general formulation of the magnetic neutron scattering cross-section [2] is somewhat more complicated, particularly when the spin polarisation of the neutron has to be accounted for. For an unpolarised beam the use of the linear response theory provides a formulation via the magnetization, which is the response to an applied magnetic perturbation. The relaxation function is

so that

$$
\phi^{\alpha \beta}(l, t)=\left(g \mu_{\mathrm{B}}\right)^{2} \frac{\mathrm{i}}{\hbar}\left\langle\left[\widehat{S}_{l}^{\alpha}(t), \widehat{S}_{0}^{\beta}(0)\right]\right\rangle,
$$

$$
\phi_{q}^{\alpha \beta}(t)=\frac{\left(g \mu_{\mathrm{B}}\right)^{2}}{N} \frac{\mathrm{i}}{\hbar}\left\langle\left[\widehat{S}_{q}^{\alpha}(t), \widehat{S}_{-q}^{\beta}(0)\right]\right\rangle .
$$

The dynamical magnetic susceptibility is then

$$
\chi_{\boldsymbol{q}}^{\alpha \beta}(\omega)=\int_{0}^{\infty} \mathrm{d} t \exp (\mathrm{i} \omega t) \phi_{\boldsymbol{q}}^{\alpha \beta}(t)
$$

which yields 


$$
\begin{aligned}
& \frac{\mathrm{d}^{2} \sigma}{\mathrm{d} \Omega \mathrm{d} E^{\prime}}=\left(\gamma r_{0}\right)^{2}\left[\frac{1}{2} g F(\boldsymbol{Q})\right]^{2} \frac{k_{\mathrm{f}}}{k_{\mathrm{i}}} \exp [-2 W(Q)] \\
& \quad \times \frac{N}{\pi\left(g \mu_{\mathrm{B}}\right)^{2}} \frac{\exp \left(\hbar \omega / k_{\mathrm{B}} T\right)}{\exp \left(\hbar \omega / k_{\mathrm{B}} T\right)-1} \sum_{\alpha \beta}\left(\delta_{\alpha \beta}-\widetilde{Q}_{\alpha} \widetilde{Q}_{\beta}\right) \operatorname{Im} \chi_{q}^{\alpha \beta}(\omega),
\end{aligned}
$$

where $r_{0}=2.818 \times 10^{-15} \mathrm{~m}$ is the classical electron radius, $\gamma=-1.913$ is the gyromagnetic ratio of the neutron, $g-$ the Landé factor, $Q=k_{\mathrm{i}}-k_{\mathrm{f}}, \widetilde{Q}=Q /|Q|$ and $F(Q)$ is the magnetic form factor, which depends on the precise magnetic properties of the system. For specific cases, the first step is to determine the relevant operator to describe the spin system. Classical cases are localized magnetism of insulators, itinerant magnetism of metals, and rare-earth magnetism.

For an ordered magnetic system, this expression may be simplified to get a scattering cross-section for spin waves somewhat similar to that of phonons, where the strength of the scattering is now related to the magnetization. For instance, in the case of collinear localized electronic spins, the scattering law for spin waves may be written as

$$
\begin{aligned}
& S(\boldsymbol{Q}, \omega) \propto\left(1+\widetilde{Q}_{\zeta}^{2}\right) \sum_{\tau} \delta(\boldsymbol{Q}-\boldsymbol{q}-\boldsymbol{\tau}) \\
& \quad \times \sum_{j}\left|\sum_{l} F_{l}(\boldsymbol{Q}) \sqrt{S_{l}} \exp \left(-\boldsymbol{\tau} \cdot \boldsymbol{r}_{l}\right) t_{l j}(q)\right|^{2}\left\{n\left[\omega_{j}(\boldsymbol{q}), T\right]+1\right\} \delta\left[\omega-\omega_{j}(q)\right],
\end{aligned}
$$

where $\zeta$ is the direction of the electronic spins, $l$ is the index of the different spins, at position $r_{l}$ in the primitive cell and $j$ is the label of spin-wave modes, $t_{l j}(q)$ are the elements which define the change of variables used to diagonalize the Hamiltonian of the magnetic system.

The measurement of the spin-wave spectrum allows the determination of exchange integrals between magnetic ions, as well as the anisotropy giving rise to a gap at $\boldsymbol{q}=0$. But more generally the dynamical magnetic response of a system may provide very interesting information on the involvement of magnetic characteristics in the properties of a solid. The case of high- $T_{\mathrm{c}}$ superconductors is a very interesting example of that kind.

\subsection{Magnetic excitations in the spin-Peierls compound $\mathrm{CuGeO}_{3}$}

In Fig. 5 are reported the magnetic excitations observed in $\mathrm{CuGeO}_{3}$ [10]. This compound is the first inorganic one where a spin-Peierls transition has This transition is due to a spin-lattice coupling, which prevents the onset of an antiferromagnetic order of the chains of spin $1 / 2 \mathrm{Cu}^{2+}$ ions imbedded in the structure. Indeed, the coupling favours a dimerization between adjacent $\mathrm{Cu}$ ions, which induces a structural transition with a non-magnetic ground state. The magnetic excitations are then excitations between the singlet ground state and the first excited triplet state. The dispersion reported in Fig. 5 reflects their $q$-dependence due to first and second nearest neighbours magnetic interactions along the chain direction $c$. The calculated curve uses the approximate relation

$$
\omega=\sqrt{\Delta^{2}+\left(\omega_{M}^{2}-\Delta^{2}\right) \sin ^{2}\left(2 \pi q_{c}\right)},
$$




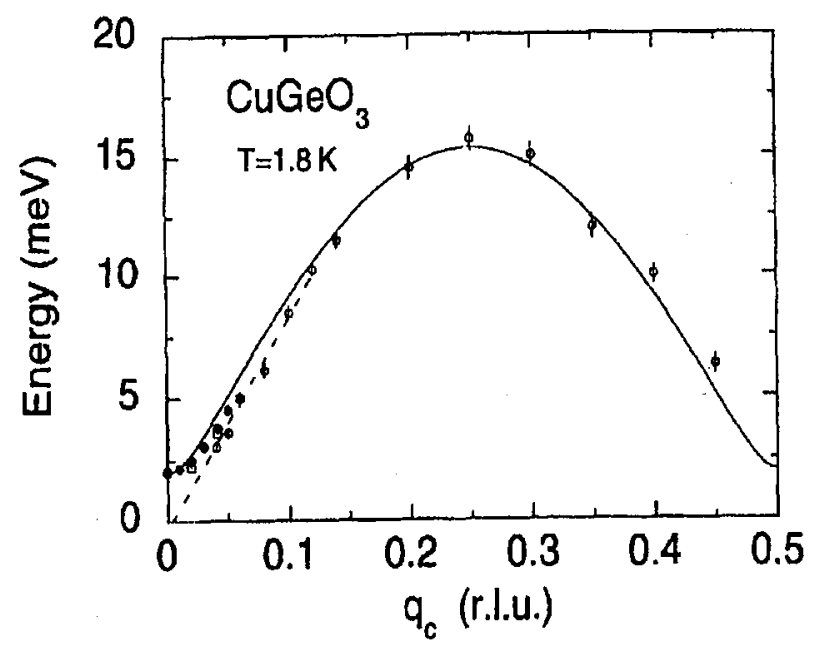

Fig. 5. Dispersion of the magnetic excitations at $1.8 \mathrm{~K}$ along $c^{*}$ in $\mathrm{CuGeO}_{3} . q_{\mathrm{c}}$ is in reduced lattice unit (r.l.u.) so that $q\left(\AA^{-1}\right)=q_{c} 2 \pi / c$. The spin-Peierls transition takes place at $T_{\mathrm{SP}}=14.2 \mathrm{~K}$. The solid line corresponds to the calculation [10].

where $\Delta \approx 1.05\left(2 J_{1}\right)(1-\alpha)^{2 / 3}$ and $\omega_{M} \approx \pi J_{1}(1+\alpha) / 2$, and $\alpha=J_{2} / J_{1}$ is the alternation parameter induced by the lattice distortion. The measurement along $a^{*}$ and $b^{*}$ gave the interchain couplings.

Actually, this is only a part of the magnetic excitations of such a low-dimensionality system. A continuum of excitations, predicted for 1D magnetic system has indeed been evidenced [11], with some peculiarities which bring a deeper insight of the magnetism of this system.

On the other hand, a soft phonon mode was expected as the structural signature of the spin-lattice coupling. A minute study of the phonon spectrum [12] demonstrated that this is not the case. After getting a detailed phonon spectrum, which allowed an adequate modelling of the lattice dynamics, a symmetry analysis was used to identify the phonons which could be involved in the transition. These phonons showed no significant softening when lowering the temperature. But the atomic displacements associated with these phonons lead to variations of the bonding angles which are involved in the magnetic superexchanges between copper ions. Therefore, the current conclusion is that a more complex dynamical magnetoelastic coupling is likely involved in the transition [12].

\subsection{Magnetic dynamical susceptibility in high- $\mathrm{T}_{\mathrm{c}} \mathrm{YBa}_{2} \mathrm{Cu}_{3} \mathrm{O}_{6+x}$}

The search for a dynamical magnetic response in high- $T_{\mathrm{c}}$ compounds has been a challenge of these last years. $\mathrm{La}_{2-x} \mathrm{Sr}_{x} \mathrm{CuO}_{4}$ and $\mathrm{YBa}_{2} \mathrm{Cu}_{3} \mathrm{O}_{6+x}$ systems gave very different results and the contribution of magnetic properties to the electron-pairing responsible for the high values of $T_{\mathrm{c}}$ in superconducting cuprates is highly debated. Triple-axis spectrometry has been determinant in this field, as the magnetic response is strongly peaked in the reciprocal space. This response has been found at antiferromagnetic (AF) positions reminiscent of the $\mathrm{AF}$ order of 

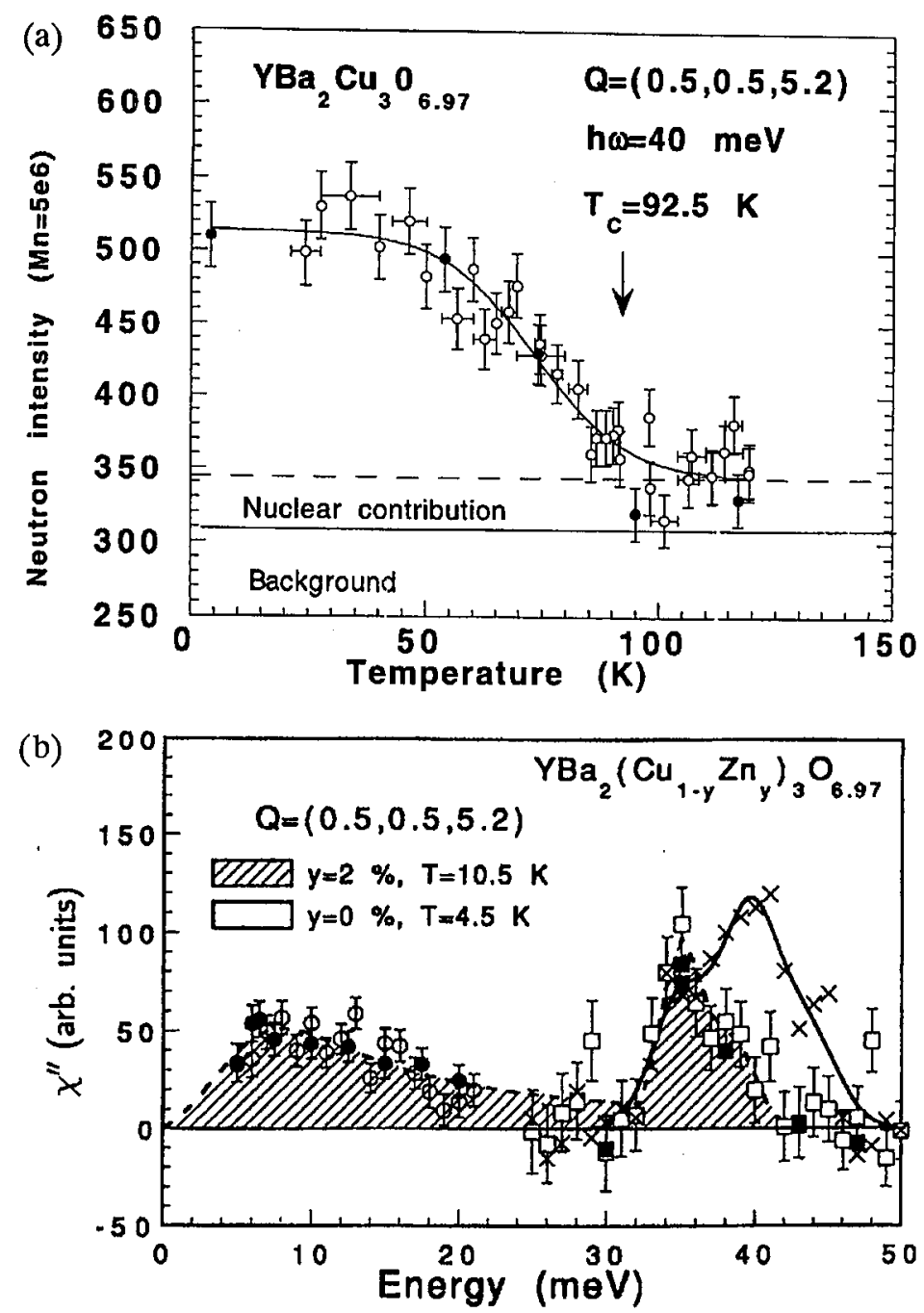

Fig. 6. (a) Temperature dependence of the neutron scattering observed at $40 \mathrm{meV}$, at antiferromagnetic position, in $\mathrm{YBa}_{2} \mathrm{Cu}_{3} \mathrm{O}_{6.97}$ [13]. Open symbols correspond to simple countings, filled symbols correspond to data obtained by a $\boldsymbol{Q}$ dependent measurement at constant energy of $40 \mathrm{meV}$. Incident neutron flux was monitored to a value $(\mathrm{Mn})$ of $5 \times 10^{6}$ (Mn corresponds to the number of incident neutrons multiplied by the monitor efficiency). The magnetic contribution to the scattering disappears at $T_{c}$, with the superconductivity. (b) Energy dependence in the superconducting phase of the dynamical magnetic response. In the pure compound (crosses) $T_{c}=92.5 \mathrm{~K}$, there is a spin gap of about $32 \mathrm{meV}$ and a resonance peak at $40 \mathrm{meV}$. In the substituted sample (circles and squares correspond to different measurements) $T_{\mathrm{c}}=69 \mathrm{~K}$, low-energy excitations filled in the gap and the resonance peak disappeared [14]. Open and filled symbols have the same meaning as in (a). 
the insulating parent compounds, in $\mathrm{YBa}_{2} \mathrm{Cu}_{3} \mathrm{O}_{6+x}$, and at incommensurate positions in the vicinity of this AF positions in $\mathrm{La}_{2-x} \mathrm{Sr}_{x} \mathrm{CuO}_{4}$. The very existence of such a magnetic response was already quite unusual as the magnetic response of unpaired electrons in normal metal is so tiny and smeared out in $q$-space that it is not experimentally sizeable. But even more, in $\mathrm{YBa}_{2} \mathrm{Cu}_{3} \mathrm{O}_{6+x}$ system a part of this dynamical magnetic response, near $40 \mathrm{meV}$, has been recognized as directly related to superconductivity. This is illustrated in Fig. 6a, where the temperature dependence of the so-called resonance in $\mathrm{YBa}_{2} \mathrm{Cu}_{3} \mathrm{O}_{6.97}$ [13] is reported, and in Fig. $6 \mathrm{~b}$, through the effects induced by zinc substitution of copper in the $\mathrm{CuO}_{2}$ planes, which affects drastically the superconductivity [14]. Furthermore, it has been shown very recently [15], again with inelastic neutron scattering, that such a dynamical magnetic response does also exist in $\mathrm{Bi}_{2} \mathrm{Sr}_{2} \mathrm{CaCu}_{2} \mathrm{O}_{8+\delta}$. This strengthens the idea that this peculiar dynamical magnetic response is a general feature of the copper oxide superconductors and that it should be taken as a determinant check for testing the validity of any proposed mechanism for the high- $T_{\mathrm{c}}$ superconductivity.

\section{References}

[1] B. Dorner, Acta Crystallogr. A 28, 344 (1972).

[2] S. Lovesey, Theory of Neutron Scattering from Condensed Matter, Oxford University Press, Fairlawn (NJ) 1984.

[3] B. Prevot, B. Hennion, B. Dorner, J. Phys. C, Solid State Phys. 10, 3999 (1977).

[4] B. Hennion, B. Prevot, M. Krauzman, R.M. Pick, B. Dorner, J. Phys. C, Solid State Phys. 12, 1609 (1979).

[5] Cheng-Zhang Wang, Rici Yu, H. Krakauer, Phys. Rev. Lett. 72, 368 (1994).

[6] J.P. Pouget, B. Hennion, C. Escribe-Filippini, M. Sato, Phys. Rev. B 43, 8421 (1991).

[7] B. Hennion, J.P. Pouget, M. Sato, Phys. Rev. Lett. 68, 2374 (1992).

[8] E. Tutis, S. Barisic, Phys. Rev. B 43, 8431 (1991).

[9] G. Travaglini, I. Morke, P. Wachter, Solid State Commun. 14, 1037 (1974).

[10] L.P. Regnault, M. A ïn, B. Hennion, G. Dhalenne, A. Revcoleschi, Phys. Rev. B 53, 5579 (1996).

[11] M. Arai, M. Fujita, M. Motokawa, J. Akimitsu, S.M. Bennington, Phys. Rev. Lett. 77, 3649 (1996).

[12] M. Braden, B. Hennion, W. Reichardt, G. Dhalenne, A. Revcoleschi, Phys. Rev. Lett. 80, 3634 (1998).

[13] P. Bourges, L.P. Regnault, Y. Sidis, C. Vettier, Phys. Rev. B 53, 876 (1996).

[14] Y. Sidis, P. Bourges, B. Hennion, L.P. Regnault, R. Villeneuve, G. Collin, J.F. Marucco, Phys. Rev. B 53, 6811 (1996).

[15]' H.F. Fong, P. Bourges, Y. Sidis, L.P. Regnault, A. Ivanov, G.D. Gu, N. Koshizuka, B. Keimer, Nature 398, 588 (1999). 